\title{
Automatic Stance-Swing Phase Detection from Accelerometer Data for Peroneal Nerve Stimulation
}

\author{
ANTOON TH. M. WILLEMSEN, FEDDE BLOEMHOF, AND HERMAN B. K. BOOM
}

\begin{abstract}
The development of implantable peroneal nerve stimulators has increased interest in sensors which can detect the different phases of walking (stance and swing). Accelerometers, having a potential for implantation, are studied as detectors for the swing phase of walking to replace footswitches. Theoretically, we could show that accelerometers can be used to distinguish between stance and swing phase. Attaching accelerometers between ankle and knee joint the equivalent acceleration of the ankle joint was calculated. This resulted in a typical and reproducible signal in which the different walking phases were identified. Automatic detection algorithms, based on cross correlation calculation were developed and tested. Measurements from four healthy and four hemiplegic subjects resulted in a total of 317 and 272 steps, respectively. One of the hemiplegic subjects was considered to be a failure due to large disturbances in the acceleration signal during the swing phase of walking, which may be related to the use of crutches. Taking part of the data as a learning set and the other part as an evaluation set we found two errors in the push-off detection for both the healthy subjects and the remaining three hemiplegic subjects, out of 152 and 106 steps, respectively. In addition, we showed that when using one accelerometer closely below the knee joint almost identical results can be achieved. This could lead to a combination of sensor and stimulator into one implantable device.
\end{abstract}

\section{INTRODUCTION}

CINCE the first experiments to correct footdrop by elecStrical stimulation of the peroneal nerve [4] this technique has developed to a point where, for a selected group of patients, it can be used as an almost routine therapy. Many of the problems remaining with today's peroneal stimulators as reported [11], [13], [16] can be divided in four groups.

- Nonoptimal specificity and reliability of stimulation. This can result in excessive inversion or eversion of the foot during dorsiflexion while the amount of dorsiflexion itself is variable.

- Difficult attachment. Although the use and fixation of the peroneal stimulator is fairly straightforward, accurate electrode placement can be cumbersome especially for patients with impaired motor functions.

- Insufficient reliability. Both footswitches and the wire connecting the footswitch and stimulator are, despite continuous effort, susceptible to mechanical failure.

Manuscript received July 20, 1989; revised February 6, 1990

The authors are with the Biomedical Engineering Division, Department of Electrical Engineering, University of Twente, $7500 \mathrm{AE}$ Enschede, The Netherlands.

IEEE Log Number 9038988.
- Acceptance problems. It is found that for some patients the use of a peroneal stimulator is discontinued because of physical (pain), esthetic or psychological motives ("gadget intolerance").

Some of these problems may be solved by using a fully implantable peroneal stimulator for which first trials have been reported [9], [14]. However, in these trials only the stimulator itself was implanted whereas the power supply and the detector were still external. Conventionally the intention of a patient with footdrop to initiate a step is detected by a footswitch with heel-off as intention criterion. Footswitches, however, lack mechanical robustness, while their potential for further improvement (size, handling, and implantability) is limited. In this paper we study the possibilities of accelerometers as detectors for the swing phase to replace footswitches. Accelerometers have the potential of implantation.

\section{Methods \\ Theory \\ Considering rigid-body dynamics, an idealized ball-} and-socket ankle joint and using a seismic accelerometer attached to the lower leg the acceleration signal as measured by an accelerometer, called equivalent acceleration, is given by [15] (Fig. 1):

$$
\vec{a}_{r}=\vec{g}-\ddot{\vec{R}}-r \vec{\omega} \times\left(\vec{\omega} \times \vec{e}_{r}\right)-r \dot{\vec{\omega}} \times \vec{e}_{r}
$$

with

$\vec{a}_{r}$ Equivalent acceleration at a distance $r$ from the ankle joint.

$\vec{g}$ Gravitational acceleration.

$\ddot{\vec{R}} \quad$ Linear acceleration of the ankle joint.

$r$ Distance from the ankle joint to the sensor.

$\vec{\omega} \quad$ Angular velocity of the lower leg.

$\vec{e}_{r}$ Unity direction vector from ankle joint to sensor.

For the ankle joint (assuming $r=0$ ) this simplifies to

$$
\vec{a}_{0}=\vec{g}-\ddot{\vec{R}}
$$

Obviously $\ddot{\vec{R}} \simeq \overrightarrow{0}$ during the stance phase and $\ddot{\vec{R}} \neq \overrightarrow{0}$ during the swing phase of walking so by calculating the modulus of the equivalent acceleration $\left(\left|\vec{a}_{0}\right|\right)$ we should be able to distinguish between the stance and swing phase. 


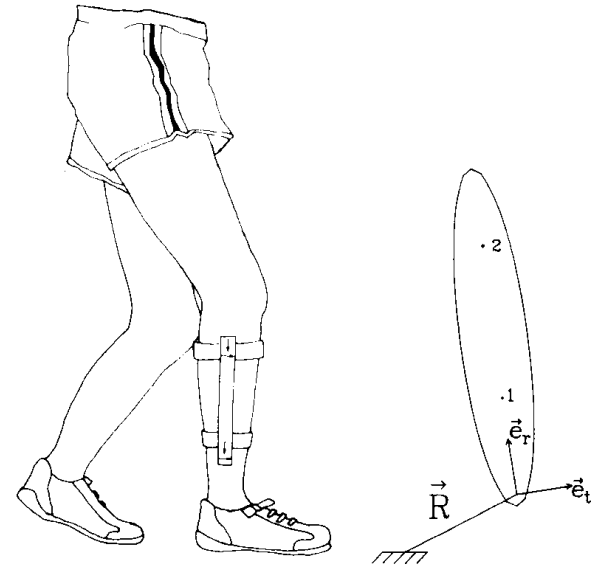

Fig. 1. Schematic representation of the measurement setup. Four accelerometers represented by arrows are attached to a bracket at positions 1 and 2 , which itself is attached to the lower leg using Velcro straps. $\vec{R}$ denotes the distance of the ankle joint to an inertial reference system, while $\vec{e}_{r}$ and $\vec{e}_{t}$ denote the unity vectors in the radial and tangential direction, respectively.

$$
\begin{aligned}
\left|\vec{a}_{0}\right| & \simeq g(\text { stance }) \\
& \neq g(\text { swing }) .
\end{aligned}
$$

Instead of placing an accelerometer at the ankle joint, which might be difficult for implantations, the equivalent acceleration can be calculated also by placing two accelerometers between the ankle and knee joint using [15]

$$
\vec{a}_{0}=\vec{g}-\ddot{\vec{R}}=\frac{r_{2} \vec{a}_{1}-r_{1} \vec{a}_{2}}{r_{2}-r_{1}}
$$

with $\vec{a}_{1}, \vec{a}_{2}$ the equivalent accelerations measured at distances $r_{1}$ and $r_{2}$ from the ankle joint, respectively.

\section{Measurements}

Because the movements of the lower extremities during walking are mainly in the sagittal plane only the tangential $\left(a_{r}^{t}\right)$ and radial $\left(a_{r}^{r}\right)$ components were measured. Therefore, four one-dimensional accelerometers (Kyowa AS-5G, size $1 \mathrm{~cm}^{3}$, weight $\pm 20 \mathrm{~g}$ ) were attached to a PVC bracket which was then attached to the lower leg using Velcro straps (Fig. 1). Footswitches were placed under the shoe at the heel and the first metatarsal head to detect the various walking phases. The signals were amplified, low-pass filtered $(100 \mathrm{~Hz})$ and sampled at $500 \mathrm{~Hz}$ for $10 \mathrm{~s}$. Measurements were recorded using a VME-based Motorola computer system (SYS133) while analysis was performed on a HP9000-300 computer. First, measurements were made with a group of healthy subjects to establish a "normal" equivalent acceleration pattern. A total of 54 measurements each $10 \mathrm{~s}$ long were made. This resulted in 317 steps which had to be detected automatically. Subjects were asked to walk at three speeds, slow, comfortable and fast. Step rate (mean \pm standard deviation) was $90 \pm 17 \mathrm{steps} / \mathrm{min}$ (range 45 to 129 ). The same measurements were done on four hemiplegic subject. Two of the hemiplegic subjects were slow walkers with walking speeds of $0.4-0.5 \mathrm{~m} \mathrm{~s}^{-2}$, one of them using two crutches. The other two hemiplegic subjects were average to good, with walking speeds of $0.8-1.0 \mathrm{~m} \mathrm{~s}^{-2}$. The accelerometers were attached to the leg also carrying the peroneal stimulator. Measurements were then performed both with and without peroneal nerve stimulation on these patients. A total of 45 measurements were made resulting in 272 steps.

\section{RESUlts}

\section{Signal Description}

In Fig. 2 a typical example of $\left|\vec{a}_{0}\right|$ is shown together with four walking phases as defined by the footswitches. Fig. 2 starts with erect standing after which the first step is performed by the leg with the attached accelerometers. The subject stopped after the last step shown. The four walking phases as defined by the footswitches are

1) Stance (heel and toe on the ground). During stance $\left|\vec{a}_{0}\right|$ approximately equals $g$ in accordance with (3).

2) Push-off (heel off the ground, toe on the ground). The push-off phase is characterized by an increase of the equivalent acceleration. A first maximum in the equivalent acceleration is within $50 \mathrm{~ms}$ of the end of the pushoff phase. Typically, the first push-off phase (standing to walking transition) is different from the following pushoff phases (Fig. 2).

3) Swing (both heel and toe off the ground). The swing phase is characterized by a down-up course of $\left|\vec{a}_{0}\right|$, ending with the heel-strike.

4) Foot-down (heel on the ground, toe off the ground). The foot-down phase starts with the heel strike which usually is clearly visible as a peak in $\left|\vec{a}_{0}\right|$. The end of the foot-down phase can not be detected directly from $\left|\vec{a}_{0}\right|$ because the ankle hardly moves during this phase $(\ddot{\vec{R}} \simeq$ $\overrightarrow{0}$ ). Typically, the last heel strike (walking to stance transition) is lower than the other heel strikes.

A peroneal nerve stimulator normally starts its stimulation in the push-off phase, detected by a heel-switch in combination with a time-delay. It ends its stimulation shortly after the heel-strike [12]. We can therefore use $\left|\vec{a}_{0}\right|$ as a trigger for a peroneal nerve stimulator provided that the push-off and the foot-down phases can be detected automatically.

To investigate the possibility of a subject independent detection criterion for the push-off phase, the last $0.4 \mathrm{~s}$. of each push-off (as defined by the toe switch) was extracted from the data. This resulted in a total of 317 pushoffs from the healthy subjects, from which we calculated both the subject specific average push-off as well as the total average push-off. The subject specific average pushoff from one subject, plus and minus the standard deviation, together with the absolute maxima and minima are shown in Fig. 3. We found that

$$
\left(m_{i}^{j}-s_{i}^{j}\right) \leq m_{i}^{k} \leq\left(m_{i}^{j}+s_{i}^{j}\right) \quad \forall i, j, k
$$




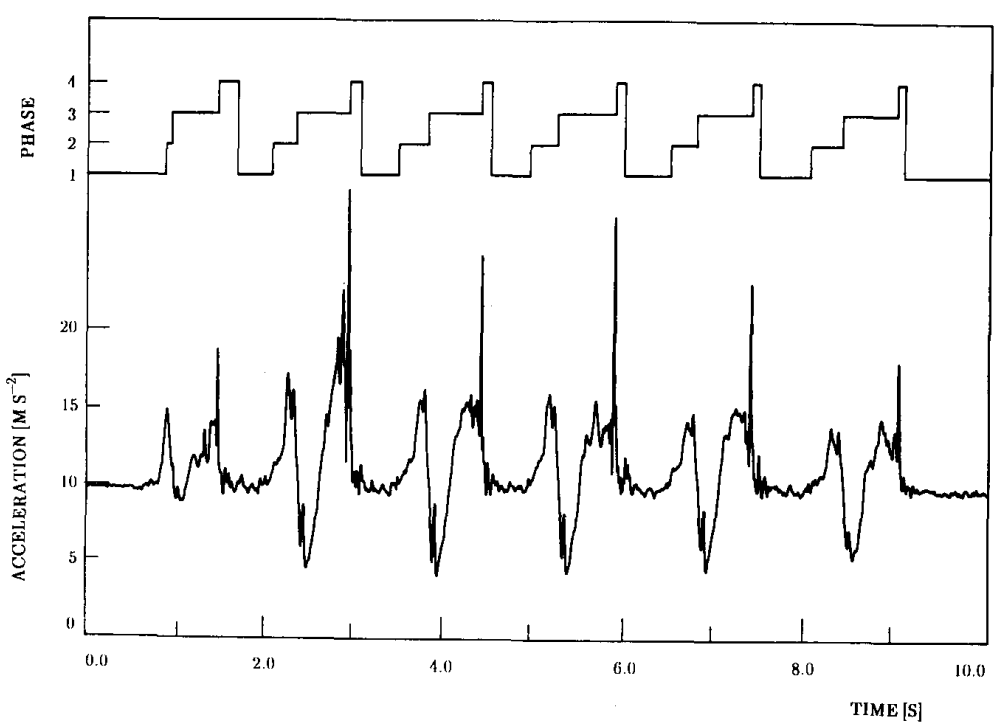

Fig. 2. Modulus of the equivalent acceleration at the ankle joint as calculated from four accelerometer signals during walking for a healthy subject in combination with a trace showing the four walking phase obtained with footswitches at heel and first metatarsal head. The subject started and ended with erect standing. The four walking are 1) stance,

2) push-off, 3) swing, and 4) footdown (see text).

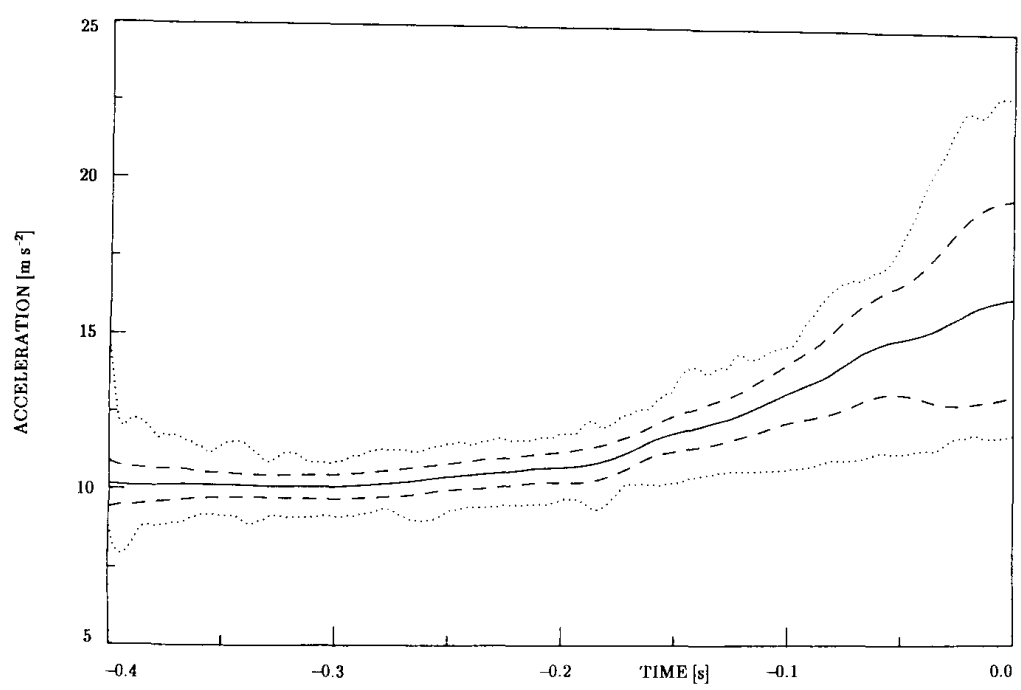

Fig. 3. Average push-off template for 1 subject with standard deviation and absolute maxima and minima (see text). The push-off ends at $t=0$. Solid line: average template. Broken line: average \pm standard deviation. Dotted line: absolute maxima and minima of the templates.

with

$m$ : average push-off.

$s: \quad$ standard deviation.

$i$ : time index.

$j, k$ : subject indexes (1..4).

which shows that the variation in the push-offs for one subject is comparable to the variation between different subjects.
We calculated the cross-correlation coefficients between the average push-off and the individual push-offs. This was done using both the subject specific average push-off as well as the total average push-off. The subject specific average push-off yielded two steps with a crosscorrelation coefficient $(r)$ below $0.9(r=0.89, r=0.73)$ while for the total average push-off only one step had a $r$ below $0.9(r=0.78)$.

The foot-down phase starts immediately after the heel 
strike. Because the heel strike can easily be recognized as a distinct peak in the acceleration signal, it was decided to use the heel strike for the detection of the foot-down phase. We found that the heel strike peak is sometimes lower than the maxima during the swing phase, so level detection can not be used. However, after differentiating the equivalent acceleration signal, a better discrimination between heel strike and the rest of the signal was possible.

The four acceleration signals from which $\left|\vec{a}_{0}\right|$, as shown in Fig. 2, was calculated are shown in Fig. 4. Comparing Figs. 2 and 4 it can be seen that the radial accelerations are very similar to $\left|\vec{a}_{0}\right|$, except for the last part of the swing phase and the heel strike. These are lower as compared to $\left|\vec{a}_{0}\right|$. In contrast, the tangential accelerations are not very similar to $\left|\vec{a}_{0}\right|$. Due to the large gravitational component, the equivalent radial acceleration $\left(a_{r}^{r}\right)$ is larger than the equivalent tangential acceleration $\left(a_{r}^{t}\right)$. As a result we find $\left|\vec{a}_{0}\right|=\sqrt[3]{\left(a_{0}^{r}\right)^{2}+\left(a_{0}^{t}\right)^{2}}$ $\approx a_{0}^{r}$. Furthermore, the equivalent radial acceleration is almost independent of the distance (Fig. 4). So, the equivalent radial acceleration as measured closely below the knee $\left(a_{2}^{r}\right)$ is a good estimate of $\left|\vec{a}_{0}\right|$. Considering this similarity between $\left|\vec{a}_{0}\right|$ and $a_{2}^{r}$ we decided to compare the result of an automatic stance-swing phase detector based on either signal.

The acceleration signals of three of the four hemiplegic subjects [Fig. 5(a)], either with or without peroneal nerve stimulation, are almost identical with the signals from the healthy subject, except for the maxima of the heel strike peaks which are higher for the hemiplegic subjects. The fourth subject showed large disturbances during the swing phase, very similar to the heel strike [Fig. 5(b)] which may be linked to his use of crutches.

\section{Detection Algorithm}

The accurate detection of the push-off and the heel strike is essential for the performance of a peroneal nerve stimulator. Because of the time critical character of the detection, i.e., the push-off must be detected as soon as possible, we decided for a syntactic detection approach [2]. One of the characteristics of this method is the segmentation of the signal (a step) in to small units (stance, pushoff, swing, foot-down). Each unit is then to be detected by its own detection algorithm. Another characteristics of this method, which is of special importance in our application, is that each unit can be detected before the end of the whole signal is reached. This is essential as the pushoff phase must be detected as soon as possible to start the stimulation.

By including a detection algorithm for the descending and ascending slopes of the swing-phase the change of a false push-off or heel strike detection during this phase can be minimized. To correct false detections, causing, e.g., stimulation during stance phase, as fast as possible, correction routines, as described below, were included. This gives the state transition diagram as shown in Fig. 6 with five normal change-state paths and three correction paths. The following algorithms are used to detect the different transitions.

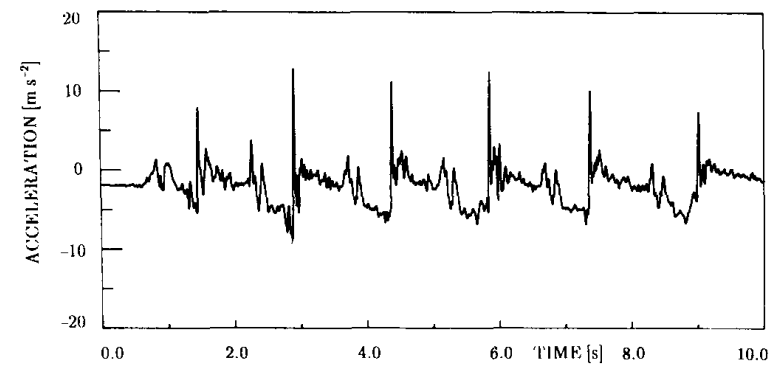

(a)

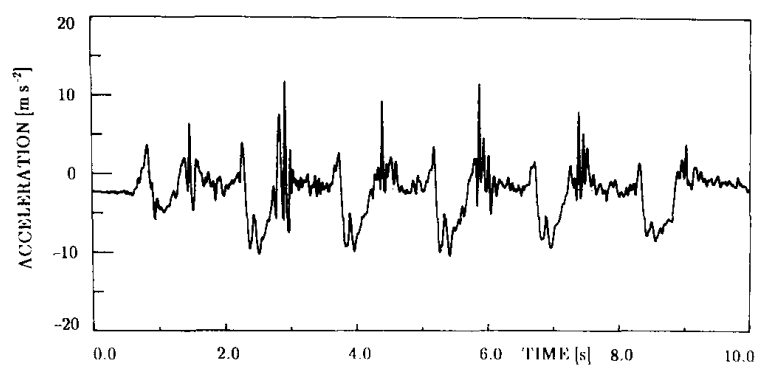

(b)
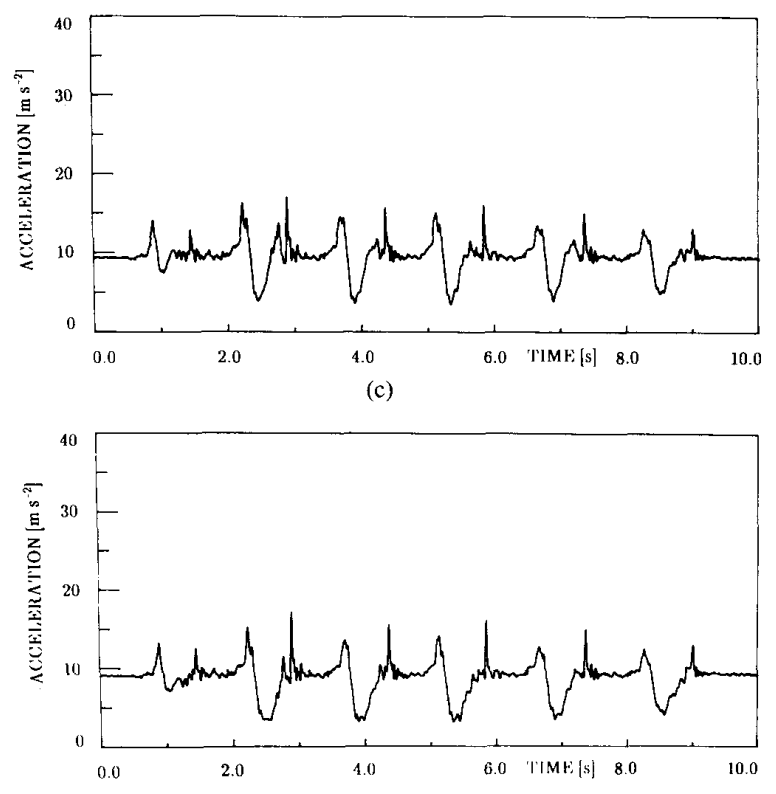

(d)

Fig. 4. Equivalent accelerations measured at different position of the lower leg during walking. (a) Tangential component, above ankle $\left(a_{1}^{t}\right)$. (b) Tangential component, below knee $\left(a_{2}^{t}\right)$. (c) Radial component, above ankle $\left(a_{1}^{r}\right)$. (d) Radial component, below knee $\left(a_{2}^{r}\right)$.

Push-Off Detection: We used the high cross-correlation coefficient between the different push-off time courses and the average push-off time course for the detection of the different push-offs. Because the cross correlation coefficient uses normalized signals (mean and standard deviation), disturbances with a comparable form as a push-off may lead to a false detection. This was prevented by including detection criteria for the mean and the standard deviation. So, for a successful push-off detection, all three 


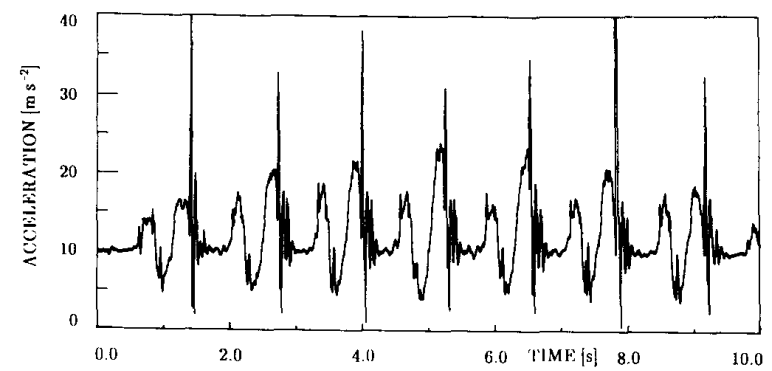

(a)

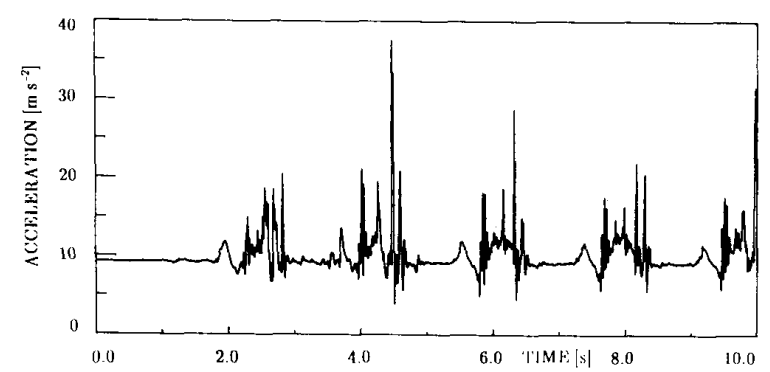

(b)

Fig. 5. Modulus of the equivalent acceleration at the ankle joint as calculated from four accelerometer signals during walking for a hemiplegic subject. (a) Typical example for three out of four subjects. (b) Typical example for fourth subject showing large disturbances during the swing phase of walking.

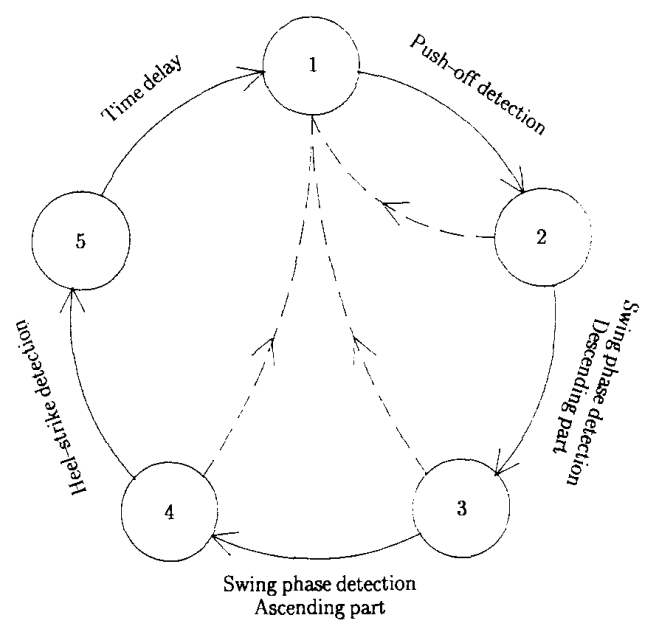

Fig. 6. Schematic diagram for a state-spcae controller showing five normal transitions and three correction paths (see text).

parameters, mean, standard deviation and correlation, had to exceed minimal levels. After a successful push-off detection we waited until the push-off detection failed, indicating the end of the push-off phase.

Swing Detection: As mentioned in the signal description the swing phase is characterized by a down-up course. The down and up course are assumed to be linear for some part which was detected by calculating the cross correlation coefficient with an arbitrary, but sufficiently long linear template. After inspection of the acceleration signal we decided for templates with slope +1 and -1 lasting
$0.2 \mathrm{~s}$ each. If detection had not been achieved within 0.5 $\mathrm{s}$ after detection of the push-off maximum, the push-off detection was assumed to be false and we returned to the stance phase. After a successful detection of the descending slope we immediately started searching for the ascending slope. This had to be accomplished within $0.5 \mathrm{~s}$ or an automatic return to stance phase was made.

Foot-Down Detection: As described before, the start of the foot-down phase can be detected from the time derivative of the heel-strike signal. This time derivative was calculated using three consecutive points $\left(a_{t}^{\prime}=\left(a_{t+1}\right.\right.$ $\left.\left.a_{t-1}\right) / 2 \Delta t\right)$ and level detection of the derivative was used to distinguish between heel-strike and noise. This had to be detected within $0.6 \mathrm{~s}$. or an automatic return to stance phase was made.

Stance Detection: Because the transition of foot-down to stance can not be detected directly from the acceleration signal (see signal description) stance was assumed to begin $0.25 \mathrm{~s}$ after the foot-down detection. This ends the stimulation. The accurate timing of the end of stimulation is a parameter for patient comfort, so the accurate detection of the heel strike is important.

\section{Accuracy of Detection}

Five parameters were optimized, as described below, to obtain maximum accuracy of detection. These parameters were, minimum value for both the cross-correlation coefficient $\left(r_{p}\right)$ and mean $\left(m_{p}\right)$ during the push-off detection, minimum value for the cross-correlation coefficient during the swing phase detection (for both the descending $\left(r_{d}\right)$ and ascending $\left(r_{a}\right)$ phase) and the minimum value for the time derivative $(h)$ for the heel-strike detection. Half of the measurements were used as a reference database to optimize the parameters and to establish a template for the push-off detection (i.e., the average push-off time course) while the other half were used for evaluation. The equivalent acceleration signal used was either $\left|\vec{a}_{0}\right|$ or $a_{2}^{r}$, i.e., the equivalent radial acceleration as measured closely below the knee joint. The template needed for the push-off detection was either the subject-specific or the total average push-off (using the reference data only). This gave a total of four combinations to be tested. The subject independent template from the healthy subjects was used for the hemiplegic subjects also. To optimize the detection we first calculated the values necessary to detect all phases of the reference data accurately. This set introduced a number of false detections. Inspection of the errors resulted in a set of detection parameters required to pretend a false detection. The final set of detection parameters was then chosen such that the number of errors, either missed detections or false detections, reached a minimum. This set was then applied to the evaluation data.

Five kind of errors occurred. First of all the controller could get "out of phase," i.e., the second part of the swing phase was detected as being the push-off and descending slope, while the push-off phase was detected as being the ascending slope and heel strike. Secondly, a step could be missed completely (no detection) or detected too 


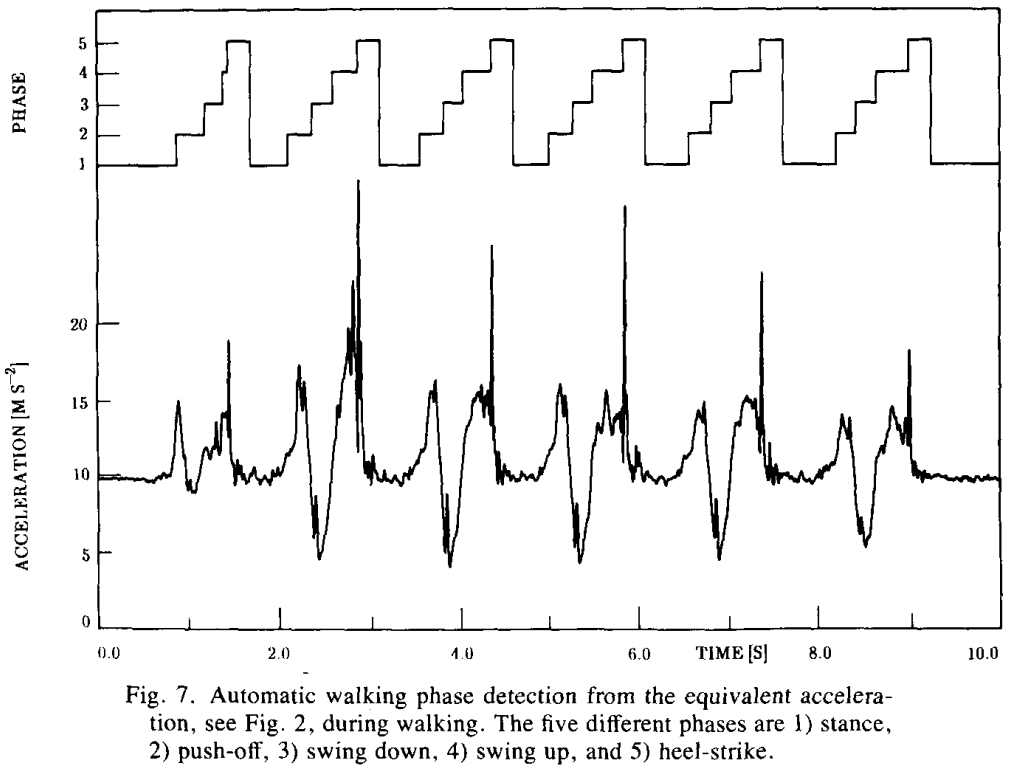

early while the end was still detected correctly (early detection). Finally, the heel strike could be missed (no heel strike) or detected to early (early heel strike). A step was considered detected accurately (no error) only if all five phases were correctly detected. Eight measurements of the healthy subjects contained a right turn. We found this to be detected as a step or not be detected at all. Fig. 7 shows a typical result with the different transitions. The results for the four healthy subjects and three of four hemiplegic subjects, with the different combinations of input signal and push-off templates are summarized in Table I using subject independent detection criteria.

The chance of a missed heel-strike detection was largest for slow walking while the chance of an early heel strike detection was largest for fast walking. The missed detection occurred at normal walking. The mean time from heel-off to toe-off, as measured with the footswitches, was $0.22 \pm 0.06 \mathrm{~s}$ while the time from heel-off to push-off detection was $0.09 \pm 0.11 \mathrm{~s}$. Time between heel contact and heel strike detection was $-0.03 \pm 0.06$ s. Results could only slightly be improved by using subject dependent detection criteria. For the hemiplegic subjects this resulted in one early detection and one missed heel strike, based on $\left|\vec{a}_{0}\right|$, and one missed detection and one missed heel strike, based on $a_{r}$. The fourth hemiplegic subject was considered a failure. The disturbances during the swing phase as shown in Fig. $5(\mathrm{~b})$, resulted either in a much to early heel-strike detection or even in a missed detection of the ascending part of the swing phase. Because there is no apparent difference between the disturbances and the actual heel-strike signal this problem can only be solved by using timing information of the different swing phases. In practice, this would mean that the push-off detection would be used to start the stimulation which would then remain on for a fixed amount of time.
TABLE I

ResUlts FOR THE AUTOMATIC DETECTION OF THE BEgINNING AND END OF A Step USing ACCElerometer Data for Four Healthy SUbject and Three of the Four Hemiplegic Subjects, Using Subject Independent Detection Parameters (See Text)

\begin{tabular}{lcccccc}
\hline $\begin{array}{l}\text { Subjects } \\
\text { Steps }\end{array}$ & \multicolumn{3}{c}{$\begin{array}{c}\text { Healthy } \\
\text { Subject }\end{array}$} & \multicolumn{3}{c}{$\begin{array}{c}\text { Hemiplegic } \\
106\end{array}$} \\
$\begin{array}{l}\text { Template } \\
\text { Source }\end{array}$ & $\left|\vec{a}_{0}\right|$ & $a_{2}^{r}$ & $\left|\vec{a}_{0}\right|$ & $a_{2}^{r}$ & $\left|\vec{a}_{0}\right|$ & $a_{2}^{r}$ \\
Parameter set & $a$ & $b$ & $a$ & $b$ & $c$ & $d$ \\
\hline Errors & & & & & & \\
$\quad$ Out of phase & 1 & 0 & 1 & 0 & 0 & 1 \\
$\quad$ No detection & 1 & 1 & 1 & 1 & 1 & 1 \\
$\quad$ Early detection & 0 & 0 & 0 & 0 & 1 & 0 \\
$\quad$ No heel strike & 0 & 3 & 0 & 3 & 1 & 1 \\
$\quad$ Early heel strike & 3 & 3 & 3 & 3 & 0 & 0 \\
$\quad$ Turning & & & & & & - \\
$\quad$ Detection & 2 & 2 & 2 & 1 & - & - \\
$\quad$ No detection & 6 & 6 & 6 & 7 & - & - \\
\hline
\end{tabular}

Set ${ }^{a} r_{p}=0.85, m_{p}=10, r_{d}=r_{a}=0.65, h=200$

${ }^{b} r_{p}=0.75, m_{p}=9.9, r_{d}=r_{a}=0.65, h=100$

${ }^{r} r_{p}=0.80, m_{p}=10, r_{d}=r_{a}=0.65, h=500$

${ }^{d} r_{p}=0.70, m_{p}=9.9, r_{d}=r_{a}=0.65, h=250$

\section{Discussion}

Conventionally the intention of a patient to initiate a step is detected by footswitches. Other methods being investigated for paraplegics are hand switches, EMG measurements [3], measurement of shoulder movement [6], pressure distribution under the feet [5] and crutches, vertical acceleration at the greater trochanter [10] and combinations. This can be combined with a number of position sensors (electrogoniometers or flexible goniometers) to form a rule-based detector [1], [6] to both detect the subject's intention and to insure that the initial conditions are correct before stimulation is started. If some voluntary control of the lower extremities is available, detection methods would preferably be capable of using this. Of the 
methods described this leaves the use of EMG and the lower extremity position detection as alternative step detectors to be used with a peroneal nerve stimulator.

Investigating the potential of accelerometers for the automatic stance-swing phase detection, emphasis was put on the accurate push-off detection which is essential for peroneal nerve stimulation. As shown (Table I) this resulted in only one missed detection and one early detection. Obviously, the effects of an incorrect push-off detection can be severe; however, many hemiplegics indicate an occasional malfunctioning of the heelswitch which they react to adequately. The minimal differences for the results between the subject specific and total average template indicates that an expansion of the learning set would not improve the results significantly.

We found the heel-strike detection to be somewhat less accurate. However, incorrect heel-strike detection is expected to have little effect on the walking ability of a hemiplegic subject. A missed heel-strike detection will result in the stimulation to continue in to the first part of the stance phase. Although this may be uncomfortable to the subject, this should not impede his walking ability. A similar effect is anticipated for early heel-strike detections. Provided that the stimulation is not switched off before the actual heel strike, i.e., a false detection is no more than $0.25 \mathrm{~s}$ before the actual heel strike, this will result in a too fast planar flexion of the foot only. Again, this should not impede the walking ability of the subject.

Out-of-phase errors combine the effects of a missed detection and a missed heel strike because during the begin of swing the stimulator is switched off, while it stays on during the first part of stance. Hemiplegics could solve this by waiting for $0.6 \mathrm{~s}$ to force a time out, thereby ensuring that the detector is in the stance phase before the next step is performed. It would be better though to improve the detector to minimize the chance of out-of-phase errors. This may be achieved by setting limits on the standard deviation, which is expected to differ significantly between push-off and swing phase.

For the limited number of measurements the only systematic difference between three of the four hemiplegics, either with or without stimulator, and the four healthy subjects was the higher amplitude of the heel-strike peak. The detection results for these hemiplegics were comparable with those of the healthy subjects. Using the detection method as presented, the fourth hemiplegic subject must be considered as a failure. Because two hemiplegic subjects were slow walkers, and the results for the second are comparable to the others, we conclude that walking speed alone is not a good indication for the applicability of our method. The second hemiplegic, however, had a better walking pattern, showing good foot clearance, and could walk unsupported. So, the walking pattern and especially the foot movements are a first indication for the applicability of our method for a specific subject.

An advantage of a detection using the signal of only one accelerometer, placed closely below the knee, is the possibility to integrate the sensor and stimulator into one implantable package. This prevents the need for additional vulnerable leads too. As shown, this resulted in a quality comparable with a detection using all four accelerometers, except for the number of missed heel-strike detections. We found higher heel strikes close to the ankle joint which shows that damping occurs. This may partly be caused by the mass of the bracket and the connection to the lower leg. For a small, lightweight accelerometer, a better result may be anticipated. If the sensor is to be implanted, fixation to the bone will be needed and even better results may be expected. So, eventually, we expect similar results for heel-strike detection when using only one accelerometer as compared with the use of all four.

The development of accelerometers using IC-technology [7], [8] should meet the requirements for size, weight and power consumption as well as mechanical and electrical robustness. The real-time stance-swing phase detection using cross-correlation techniques is well within the reach of today's "standard" microprocessors. Simulating a sample frequency of $100 \mathrm{~Hz}$, we established, based on the Motorola computer system (12.5 MHz, MC68020 CPU and MC68881 FPU), the time for detection at \pm 5 $\mathrm{ms}(50 \%)$. Currently, our department is involved with the development of an implantable two-channel peroneal nerve stimulator with approximate dimensions of $25 \mathrm{~mm}$ $\phi$ by $7 \mathrm{~mm}$, having a power consumption of around 20 $\mathrm{mW}$. Taking the same values for our detector we expect the size to be achievable, although the required power consumption is, to the best of our knowledge, an order of magnitude lower than achievable with present available microprocessors.

\section{REFERENCES}

[1] B. J. Andrews, R. H. Baxendale, R. Barnett, G. F. Phillips, T. Yamazaki, J. P. Paul, and P. A. Freeman, "Hybrid FES orthosis incorporating closed loop control and sensory feedback," J. Biomed. Eng., vol. 10, pp. 189-195, 1988.

[2] K. S. Fu, Syntactic Methods in Pattern Recognition. New York: Academic, 1974

[3] D. Graupe and K. H. Kohn, "A critical review of EMG-controlled electrical stimulation in paraplegics," CRC Crit. Rev. Biomed. Eng., vol. 15, pp. 187-210, 1988 .

[4] W. T. Liberson, H. I. Holmquest, D. Scott, and M. Dow, "Functional electrotherapy in stimulation of the peroneal nerve synchronized with the swing phase of the gait of hemiplegic patients,' Arch. Phys. Med. Rehab., vol. 42, pp. 202-205, 1961

[5] C. Miao, W. H. Ko, A. Massiello, and T. M. Spear, "Body position and pressure distribution sensors in prosthetic systems for computercontrolled walking," Transducers 1987, 4th Int. Conf. Solid State Sensors \& Act., Tokyo, 1987, pp. 57-60

[6] J. S. Petrofsky, C. A. Phillips, and H. H. Heaton, "Feedback control system for walking in man," Comput. Biol. Med., vol. 14, pp. 135 $149,1984$.

[7] L. M. Roylance and J. B. Angell, "A batch-fabricated silicon accelerometer," IEEE Trans. Electron. Dev., vol. ED-26, pp. 1911-1917, 1979.

[8] F. Rudolf, A. Jornod, and P. Bencze, "Silicon microaccelerometer," in Proc. Transducers 1987, 4th Int. Conf. Solid State Sensors and Actuators., Tokyo, 1987, pp. 395-398.

[9] P. Strojnik, R. Acimovic, E. Vavken, V. Simic, and U. Stanic, "Treatment of drop foot using an implantable peroneal underknee stimulator," Scand. J. Rehab. Med., no. 19, pp. 37-43, 1987.

[10] J. Symons, D. R. McNeal, R. L. Waters, and J. Perry, "Trigger switches for implantable gait stimulation," RESNA 9th Ann. Conf. Minneapolis, MN, 1986, pp. 319-321.

[11] K. Takebe, C. Kukulka, B. S. Mysore, G. Narayan, M. Milner, and J. V. Basmajian, "Peroneal nerve stimulator in rehabilitation of hemiplegic patients," Arch. Phys. Med. Rehab., vol. 56, pp. 237-240, 1975 . 
[12] A. Trnkoczy, U. Stanic, and T. Jeglic, "Electronic peroneal brace with a new sequence of stimulation," Med. Biol. Eng., pp. 570-576, 1975.

[13] R. L. Waters, D. R. McNeal, B. Clifford and W. Faloon, "Longterm follow-up of peroneal NMA patients," in Advances in External Control of Human Extremities, D. Popovic, Ed. Belgrade, pp. 471$477,1984$.

[14] R. L. Waters, D. R. McNeal, and J. Perry, "'Experimental correction of footdrop by electrical stimulation of the peroneal nerve," J. Bone Joint Surg., no. 8, pp. 1047-1054, 1975.

[15] A. Th. M. Willemsen, J. A. van Alsté, and H. B. K. Boom, "Accelerometers and functional electrostimulation," in Electro-Physiological Kinesiology., W. Wallinga, H. B. K. Boom, and J. de Vries, Eds. Amsterdam: Elsevier, 1988, pp. 105-108.

[16] G. Zilvold, "Follow up of patients with a peroneal stimulator: Indications for prescription," in Advances in External Control of Human Extremities, D. Popovic, Ed. Belgrade, 1981, pp. 245-250.

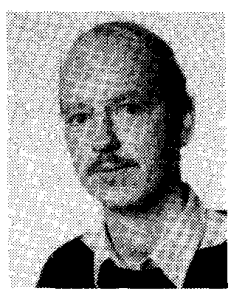

Antoon Th. M. Willemsen received the M.Sc. and $\mathrm{Ph} . \mathrm{D}$. degrees in applied physics from the University of Twente, The Netherlands, in 1984 and 1990 , respectively.

In 1985 he joined the Biomedical Engineering Division of the Department of Electrical Engineering, University of Twente, working in the field of functional neuromuscular stimulation for the rehabilitation of spinal cord injured patients. $\mathrm{His}$ research interest are sensors for gait assessment, specifically the applicability of accelerometers, on which he wrote his dissertation-accelerometers for gait assessment in functional neuromuscular stimulation.

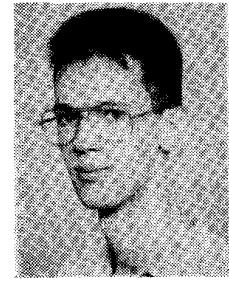

Fedde Bloemhof was born in Leeuwarden, The Netherlands, in 1963. He is currently a M.Sc. student at the Department of Electrical Engineering at the University of Twente, The Netherlands. From 1988 to 1989 he was with the Biomedical Engineering Division, University of Twente, working in the field of functional neuromuscular stimulation. His main field of interest was in the automatic stance-swing phase detection using accelerometers.

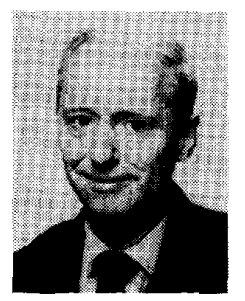

Herman B. K. Boom was trained as a medical physicist at the University of Utrecht, The Netherlands, where he received the Ph.D. degree in 1971.

He joined the Department of Medical Physics and Medical Physiology where he was engaged in research in the field of cardiac mechanics and taught physiology and biophysics. Since 1976 he has held the Chair of Medical Electronics in the Department of Electrical Engineering and is Chairman of the Biomedical Engineering Division, University of Twente, The Netherlands. His research interests are cardiovascular system dynamics, bioelectricity, and rehabilitation technology. 